\title{
Inhibited composition for work in marine conditions
}

\author{
Ekaterina Plaskeeva $^{1 *}$, Valery Trusov ${ }^{2}$ \\ ${ }^{1}$ Postgraduate Student, Assistant of the department of Chemistry, State marine technical university of Saint-Petersburg, Lotsmanskaya, 3, \\ St. Petersburg 190008, Russian Federation \\ ${ }^{2}$ The professor, Dr.Sci.Tech., the professor of department of Chemistry, State marine technical university of Saint-Petersburg, \\ Lotsmanskaya, 3, St. Petersburg 190008, Russian Federation
}

\begin{abstract}
In marine conditions, especially in the Arctic, the problems of reliability and safety of marine corrosion protection systems are relevant. Reliability can be enhanced by corrosion inhibitors. For the equipment not subject to painting, safe technologies for preservation of water-based compounds are substantiated by bioassay methods. When carrying out painting work carried out by crew members at sea, a simplified chemical surface preparation was proposed with the «NOTEH» phosphating compound with anode inhibitors. The topic of the discussion are the passivation properties of «NOTEH» according to the data on the suppression of corrosion under a layer of paint primer. To increase the safety of work, hexavalent chromium compounds were removed from the inhibiting block of «NOTEH» additives.
\end{abstract}

\section{Danger of temporary protection}

The atmospheric corrosion inhibitors are the most advanced means of "temporary" protection and the main component of preservation compounds [1]. But they may contain more toxic substances than the components of paints - nitrites, chromates, amine derivatives, etc. In order to assess the impact on the surrounding aquatic environment, we studied the degree of toxicity of aqueous solutions of inhibitors using biotesting methods, that is, using test objects that signal danger, regardless of which substances and in what combination cause changes in vital functions of the test objects. Chlorella Vulgaris Beijer test cultures were used as biosensors on Tamiya and Daphnia Magna Straus 5\% culture medium. A comparative analysis of aqueous solutions of the following corrosion inhibitors was carried out in their working concentration of $1 \%$ of the mass: H-M-1 is a complex salt of a cyclic amine and natural plant fatty acids [2]. FMT - tall oil fatty acids modified with copper derivatives of chlorophyll [3]. It is insoluble in water, but forms a stable emulsion in an alkaline solution of sodium carbonate. $\mathrm{Na}_{2} \mathrm{CO}_{3}$ and $\mathrm{K}_{2} \mathrm{Cr}_{2} \mathrm{O}_{7}$ are inorganic anode-type inhibitors for closed water systems. It was established that the toxicity of the soda emulsion of FMT and the soda solution itself are the same. The manifestation of the toxicity of FMT is limited only by the alkalinity of the sodium carbonate solution. At the same level is the toxicity of the H-M-1 solution. Thus, before the discharge of the wastes (solutions after deconservation) corrosion inhibitors N-M-1, FMT, $\mathrm{Na}_{2} \mathrm{CO}_{3}$, they must be diluted with water. Based on the data obtained and the calculation of the toxicity criterion of the samples, it was concluded that the inhibitors are classified as hazard classes 3 or 2 . For the purpose of preserving marine equipment for the period of storage, shutdown, repair, preference should be given to aqueous solutions of low-hazard N-M-1 and FMT.

\section{Improving the reliability of the paint coating system}

There are various ways to create coatings of high reliability. For example, the Belgian paint "Ecospid" [4] has the effect of a maze. The diffusion of oxygen molecules to the metal surface is hindered by glass pigment flakes. The coating thickness is about $1 \mathrm{~mm}$.

To increase the service life of the paintwork, a combination with a corrosion inhibitor can be used. Unfortunately, safe organic inhibitors are unsuitable for solving this problem, since their application to the metal contradicts the standards of cleanliness of the painted surface.

In marine conditions, the main repairs are carried out in warm enclosed spaces, tanks, compartments, where dust is dangerous during cleaning operations and toxic fumes of organic solvents of paints. We have chosen a technologically simple and universal way to combine inhibition with painting at the stage of surface preparation, taking into account the requirements of cleanliness standards [5]. For this, an inorganic watersoluble inhibitor is introduced into the phosphate conversion coating matrix. The phosphating operation has long been known and is widely used. The products of the conversion reactions - inorganic phosphates of iron, zinc, etc., possess the properties of a cathodic inhibitor due to the inhibition of oxygen access to the metal. The addition of an anodic inorganic oxidizing

\footnotetext{
$\overline{{ }^{*} \text { Corresponding author: ekaterina.plaskeeva@list.ru }}$
} 
agent contributes to the creation of a more effective inhibitory system of a mixed type of protective effect.

To protect the freeboard, superstructure and other structures operating in a marine atmosphere, we use the «NOTEH» chemical rust converter, which works in a variety of fields for the chemical preparation of metallic surfaces for painting [5]. It is a buffer mixture of phosphoric acid and zinc acid phosphates, with the addition of strong oxidizing agents. In atmospheric conditions, under the layers of paint, the inhibitors do not pollute the environment, but they can be dangerous for the crew during phosphating work. The most toxic chromates are the substances of the first hazard class. In this regard, we proposed to remove hexavalent chromium compounds from the» NOTEH» composition. To assess the effectiveness of the new additive block, sub-film corrosion was studied under a coat of paint. For testing, the epoxy ether primer EF-065 used in marine atmospheres was selected. For the tests we used samples of structural carbon steel of ordinary quality VSt3Sp2 with a size of $150 \times 70 \times 2 \mathrm{~mm}$. The surface preparation of the samples was carried out by sandblasting to the degree of Sa2 $1 / 2$ (ISO 8501-1). Three series of tests were performed, including three parallel samples. In the first series (1), one coat of primer was applied to the prepared surface. In series (2), the «NOTEH» solution was applied in 2 layers to the prepared samples with a brush. After the second layer has dried, one layer of EF-065 was applied. In the series (3), NOTEH was used with the $\mathrm{Cr}$ (VI) compounds removed. Then, all samples were dried and kept indoors for 7 days. The thickness of the primer was 27-35 microns. On the front side of each sample, a cross-shaped incision was made of the coating to the metal with a cutter in one movement along the ruler along the diagonal of the plate with a width of 0.5 $\mathrm{mm}$. After that, the samples were kept in a salt fog chamber according to GOST 9.401-91, method B (ISO 12944-6) at a temperature of $35^{\circ} \mathrm{C}$ and a concentration of sodium chloride in a continuously sprayed solution
$(50 \pm 5) \mathrm{g} / \mathrm{dm} 3$ for 240 hours. After extracting the samples from the chamber, the coating was softened with toluene and carefully removed from the samples. The value of the spread of corrosion from the notch was determined by the average value of two lines calculated taking into account the maximum damage every $10 \mathrm{~mm}$ of the notch line.

Taking into account the averaging over all three parallel samples, the results obtained are presented in the diagram. The determination of the value of the corrosion spread from the notch showed that on the samples treated with «NOTEH» it is not less than 2 times lower. Within the experimental error, the results of series (2) and (3) coincide. No negative effects of the removal of chromium compounds were found; it was compensated by a slight increase in the concentration of less toxic components of the «NOTEH» inhibitory block.

\section{Conclusion}

Thus, the atmospheric corrosion inhibitor in the conversion of the phosphate coating gives it passivating properties and inhibits corrosion under the soil layer. Without a corrosion inhibitor, the insoluble corrosion products exert pressure and damage the skeleton of the paintwork, and water-soluble salts actuate the osmotic mechanism of paint destruction. Ultimately, there is a drop in adhesion and peeling of the coating. Thanks to the inhibitor, the primary (starting) corrosion mechanisms are suppressed, thereby increasing the service life of the coating. It should be noted that the data obtained by us correlate with the conclusions of examinations and data from the operation of «NOTEH» in various climatic zones on the increase in the service

The most successful phosphating with inhibition can be used in simplified paint preparation technologies for the repair of metal structures in difficult marine conditions. The controlled operation of «NOTEH» was successfully held in the Arctic on the Prirazlomnaya

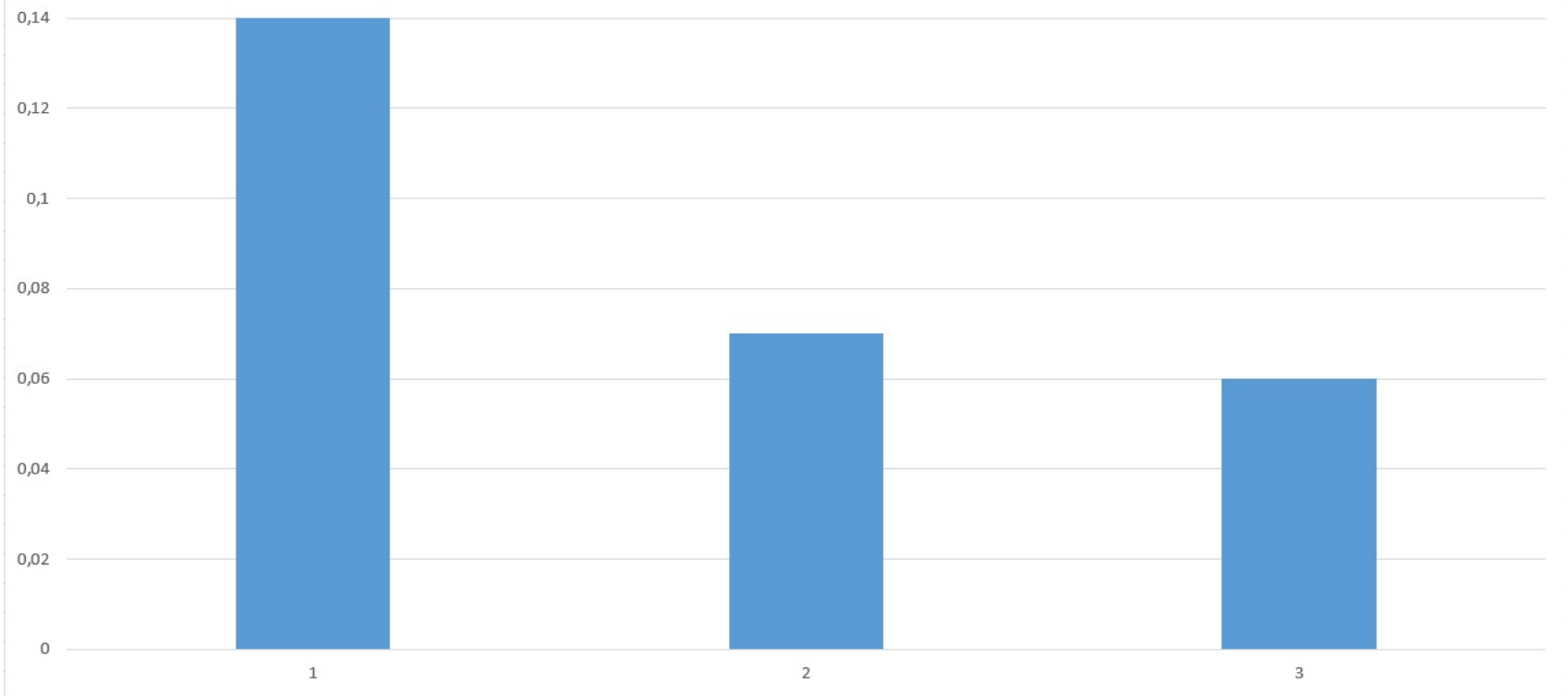


platform. The composition is recommended for further use.

\section{References}

1. I.L.Rosenfeld, V.P.Persiantseva. Inhibitors of atmospheric corrosion. M. Science. 1985. 279C.

2. R.S.Krymskaya, V.I. Trusov, A.I. Altsybeeva, T.M.Kuzinova. Corrosion Inhibitor H-M-1. Corrosion: materials, protection. 2011. №9. P.32

3. V.I. Trusov, E.A.Bezrodnykh, E.A. Nazarov, S.A. Lebedeva Corrosion inhibitor with chlorophyll copper derivatives Corrosion: materials, protection. 2004. №10 C.29-30

4. Boud Van Rompuy. "Surface-treated composites. The White Book. ".HOKAPRESS, 2012. 243-262 p.URL:

https://boudvanrompay.com/en/author/category/2/bo oks( appeal date 08.17.2018)

5. V.I.Trusov, R.S.Krymskaya, A.I.Gritskevich. Inhibition of corrosion in the paint coating system. Natural and technical sciences. 2016. №11 (101) C.262-265. 